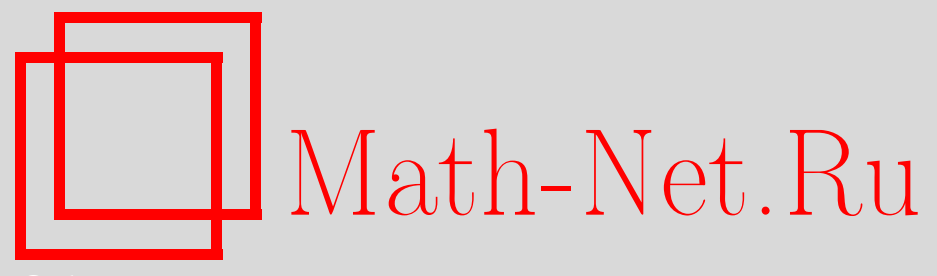

А. М. Зубков, О. К. Шибанов, Пуассоновская предельная теорема для двухэтапной равновероятной схемы размещения частиц по ячейкам, Дискрет. матем., 2006, том 18, выпуск 4, 99-104

DOI: https://doi.org/10.4213/dm74

Использование Общероссийского математического портала Math-Net.Ru подразумевает, что вы прочитали и согласны с пользовательским соглашением http://www . mathnet.ru/rus/agreement

Параметры загрузки:

IP: 107.22 .136 .117

26 апреля 2023 г., 15:11:11

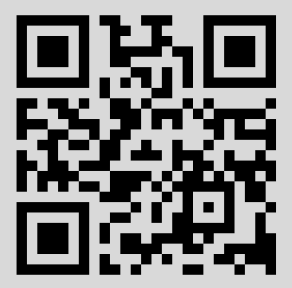




\title{
Пуассоновская предельная теорема для двухэтапной равновероятной схемы размещения частиц по ячейкам
}

\author{
๑ 2006 г. А. М. Зубков, О. К. Шибанов
}

\begin{abstract}
Рассматривается двухэтапная схема размещения частиц по ячейкам. На первом этапе $N_{0}$ исходных частиц независимо и равновероятно размещаются по $N_{1}$ ячейкам первого слоя. На втором этапе $N_{1}$ ячеек первого слоя рассматриваются как частицы и независимо и равновероятно размещаются по $N_{2}$ ячейкам второго слоя вместе с содержащимися в них исходными частицами. Указаны условия, при которых распределение числа ячеек второго слоя, содержащих ровно $r$ частиц, сходится к распределению Пуассона.

Работа выполнена при поддержке программой Отделения математических наук РАН «Современные проблемы теоретической математики» и программой Президента Российской Федерации поддержки ведущих научных школ, грант НШ 4129.2006.1.
\end{abstract}

\section{1. Введение}

Схема случайного размещения частиц по ячейкам является удобной математической моделью различных реальных процессов в физике, технике, биологии и т. п. Различные ее варианты рассматриваются в математической статистике. Предельные теоремы для ряда вариантов схемы случайного размещения частиц по ячейкам можно найти в книге [1]. В обычной схеме равновероятного размещения частищ по ячейкам предполагается, что частицы независимо одна от другой размещаются по $N$ ячейкам так, что вероятность попадания $k$-й частищы в $j$-ю ячейку равна $1 / N$ для любых $k=1, \ldots, n$ и $j=1, \ldots, N$. Пусть $\eta_{j}=\eta_{j}(n, N)$ - число частиц, попавших в $j$-ю ячейку после размещения $n$ частиц, и $\mu_{r}(n, N)$ - число ячеек, в которые попало ровно $r$ частиц. В [1] доказано, в частности, что если $r>1$ - константа, а $n, N \rightarrow \infty$ так, что

$$
\frac{1}{r !} \frac{n^{r}}{N^{r-1}} e^{-n / N} \rightarrow \lambda \in(0, \infty),
$$

то для любого фиксированного $k=0,1, \ldots$

$$
\mathbf{P}\left(\mu_{r}(n, N)=k\right) \rightarrow \frac{\lambda^{k}}{k} e^{-\lambda} .
$$

Приведенная формулировка охватывает два случая: левую область (когда $n / N \rightarrow 0$ ) и правую область (когда $n / N \rightarrow \infty$ ). 
В настоящей работе мы рассматриваем более сложную (двухэтапную, или двуслойную) схему равновероятного размещения частиц по ячейкам. Схема задается не двумя, а тремя параметрами, которые мы будем обозначать $N_{0}, N_{1}$ и $N_{2}$. На первом этапе $N_{0}$ исходных частиц независимо и равновероятно размещаются по $N_{1}$ ячейкам первого слоя. На втором этапе $N_{1}$ ячеек первого слоя рассматриваются как частицы и независимо и равновероятно размещаются по $N_{2}$ ячейкам второго слоя вместе с содержащимися в них исходными частицами. Обозначим через $\eta_{j}^{(i)}=\eta_{j}^{(i)}\left(N_{0}, N_{1}, N_{2}\right)$ число исходных частиц, попавших в $j$-ю ячейку $i$-го слоя, и через $\mu_{r}^{(i)}\left(N_{0}, N_{1}, N_{2}\right)=\mu_{r}^{(i)}$ - число ячеек $i$-го слоя, в которые попало ровно $r$ исходных частиц.

Теорема 1. Если в равновероятной двухэтапной схеме размещения частич $r>0$ фиксировано, $N_{0}, N_{1}, N_{2} \rightarrow \infty$ так, что $N_{0}=o\left(N_{2}\right), N_{0}=O\left(N_{1}\right) u$

$$
\mathbf{E} \mu_{r}^{(2)} \rightarrow \lambda \in(0, \infty) \text {, }
$$

то для любого фиксированного $k=0,1, \ldots$

$$
\mathbf{P}\left(\mu_{r}^{(2)}=k\right) \rightarrow \frac{\lambda^{k}}{k !} e^{-\lambda}
$$

Результаты анонсировались в [2, 3].

Прежде чем переходить к доказательству, отметим одну интерпретацию двухэтапной схемы равновероятного размещения частиц по ячейкам, демонстрирующую ее аналогию с процедурами, используемыми при построении оценок по методу бутстрэпа.

Сначала по обычной равновероятной схеме разместим $N_{1}$ частиц по $N_{2}$ ячейкам; пусть $\eta_{j}\left(N_{1}, N_{2}\right)$ - число частиц, попавших в $j$-ю ячейку, $j=1, \ldots, N_{2}$. Отношения

$$
\pi_{j}=\frac{\eta_{j}\left(N_{1}, N_{2}\right)}{N_{1}}, \quad j=1, \ldots, N_{2},
$$

образуют случайное распределение вероятностей. Теперь проведем размещение $N_{0}$ частиц по $N_{2}$ ячейкам в соответствии с распределением $\pi_{j}$. Нетрудно убедиться в том, что описанная схема эквивалентна двухэтапной равновероятной схеме размещения частищ. Действительно, схему размещения, в которой (случайные) вероятности попадания в ячейки выбираются в соответствии с вышеприведенной процедурой, можно описать иначе следующим образом. Первый этап размещения $N_{1}$ частиц по $N_{2}$ ячейкам соответствует случайному разбиению $N_{1}$ ячеек на $N_{2}$ подмножеств $M_{1}, \ldots, M_{N_{2}}$, содержащих $\eta_{1}\left(N_{1}, N_{2}\right), \ldots, \eta_{N_{2}}\left(N_{1}, N_{2}\right)$ элементов соответственно. Если после этого разбиения провести равновероятное размещение $N_{0}$ частиц по $N_{1}$ ячейкам, то вероятность попадания частицы в множество $M_{j}$ (и, следовательно, в $j$-ю ячейку второго слоя) будет равна $\eta_{j}\left(N_{1}, N_{2}\right) / N_{1}$.

Всюду в работе $c, c_{0}, \ldots$ обозначают некоторые положительные константы. Случайная величина $\theta_{j}=\theta_{j}(r), j=1, \ldots, N_{2},-$ индикатор события, состоящего в том, что в $j$-ю ячейку второго слоя попало ровно $r$ частиц; таким образом,

$$
\mu_{r}^{(2)}=\sum_{j=1}^{N_{2}} \theta_{j} .
$$

При натуральном $r \geqslant 2$ будем использовать обозначение

$$
K(r)=\left\{\mathbf{k}=\left(k_{1}, \ldots, k_{r}\right) \in Z_{0}^{r}: k_{1}+2 k_{2}+\ldots+r k_{r}=r\right\},
$$

где $Z_{0}=\{0,1, \ldots\}$. Число элементов множества $K(r)$ не превосходит числа целых неотрицательных решений уравнения $m_{1}+m_{2}+\ldots+m_{r}=r$, которое равно $\left(\begin{array}{c}2 r-1 \\ r\end{array}\right)$. 


\section{2. Пуассоновские предельные распределения $\mu_{r}^{(2)}$}

Нетрудно проверить, что

$$
\left\{\eta_{j}^{(2)}=r\right\}=\bigcup_{\mathbf{k} \in K(r)} C_{j}(\mathbf{k}),
$$

где $C_{j}(\mathbf{k})-$ событие, состоящее в том, что в $j$-ю ячейку второго слоя попало ровно $k_{i}$ ячеек первого слоя, содержащих ровно $i$ частиц, $i=1, \ldots, r$.

Пусть $\bar{\mu}_{1}^{(1)}=\mu_{1}^{(1)}+\ldots+\mu_{N_{0}}^{(1)}-$ число непустых ячеек первого слоя. Очевидно, что $0 \leqslant \bar{\mu}_{1}^{(1)} \leqslant \min \left(N_{0}, N_{1}\right)$.

В следующей лемме приводятся асимптотические соотношения для $\mathbf{E} \mu_{r}^{(2)}$ при некоторых способах изменения параметров $N_{0}, N_{1}, N_{2}$.

Лемма 1. В равновероятной двухэтапной схеме при любом $r \geqslant 2$

$$
\mathbf{E} \mu_{r}^{(2)}=\frac{N_{0}^{r}}{r ! N_{1}^{r-1}} e^{-N_{0} / N_{1}}\left(1+O\left(\frac{N_{1}}{N_{2}}+\frac{1}{N_{0}}+\frac{1}{N_{1}}\right)\right),
$$

если $N_{0} \rightarrow \infty, N_{0}=O\left(N_{1}\right), N_{1}=O\left(N_{2}\right)$, а если $N_{0} \rightarrow \infty, N_{0}=o\left(\min \left(N_{1}, N_{2}\right)\right)$, $N_{2}=O\left(N_{1}\right)$, mo

$$
\mathbf{E} \mu_{r}^{(2)}=\frac{N_{0}^{r}}{r ! N_{2}^{r-1}}\left(1+O\left(\frac{N_{0}}{N_{2}}+\frac{1}{N_{0}}+\frac{N_{2}}{N_{1}}\right)\right) .
$$

Доказательство. Для равновероятной двухэтапной схемы индикаторы $\theta_{1}, \ldots, \theta_{N_{2}}$, очевидно, одинаково распределены; следовательно,

$$
\mathbf{E} \mu_{r}^{(2)}=\sum_{i=1}^{N_{2}} \mathbf{P}\left(\theta_{i}=1\right)=N_{2} \mathbf{P}\left(\theta_{1}=1\right) .
$$

В силу (2) достаточно найти асимптотику $\mathbf{P}\left(\theta_{1}=1\right)$. Пусть $\mathbf{k}_{0}=(0, \ldots, 0,1)$, тогда

$$
\mathbf{P}\left(\theta_{1}=1\right)=\mathbf{P}\left(C_{1}\left(\mathbf{k}_{0}\right)\right)+\sum_{\left.\mathbf{k} \in K(r) \backslash\left\{\mathbf{k}_{0}\right)\right\}} \mathbf{P}\left(C_{1}(\mathbf{k})\right) .
$$

Вероятность отдельного события равна

$$
\mathbf{P}\left(C_{1}(\mathbf{k})\right)=\frac{\mathbf{E}\left(\mu_{1}^{(1)}\right)^{\left[k_{1}\right]} \ldots\left(\mu_{r}^{(1)}\right)^{\left[k_{r}\right]}}{k_{1} ! \ldots k_{r} ! N_{2}^{k_{1}+\ldots+k_{r}}}\left(1-\frac{1}{N_{2}}\right)^{\bar{\mu}_{1}^{(1)}-k_{1}-\ldots-k_{r}}
$$

Последний множитель (с учетом неравенства $\left.k_{1}+\ldots+k_{r} \leqslant \bar{\mu}_{1}^{(1)} \leqslant \min \left(N_{0}, N_{1}\right)\right)$ можно оценить так:

$$
1 \geqslant\left(1-\frac{1}{N_{2}}\right)^{\bar{\mu}_{1}^{(1)}-k_{1}-\ldots-k_{r}} \geqslant 1-\frac{\min \left(N_{0}, N_{1}\right)-k_{1}-\ldots-k_{r}}{N_{2}} .
$$

Согласно [1], с. 45, при $k_{1}+\ldots+r k_{r}=r$

$$
\mathbf{E}\left(\mu_{1}^{(1)}\right)^{\left[k_{1}\right]} \ldots\left(\mu_{r}^{(1)}\right)^{\left[k_{r}\right]}=\frac{N_{0}^{[r]} N_{1}^{\left[k_{1}+\ldots+k_{r}\right]}}{N_{1}^{r}(1 !)^{k_{1}} \ldots(r !)^{k_{r}}}\left(1-\frac{k_{1}+\ldots+k_{r}}{N_{1}}\right)^{N_{0}-r} .
$$


Так как $-x-x^{2}<\ln (1-x)<-x$ при $0<x<1 / 2$, то при $N_{1}>2 k$

$$
e^{-k N_{0} / N_{1}} e^{-k N_{0} / N_{1}^{2}}<\left(1-\frac{k}{N_{1}}\right)^{N_{0}-r}<e^{-k\left(N_{0}-r\right) / N_{1}}
$$

Заменяя факториальные степени обычными и учитывая условия леммы 1 , получаем:

$$
\mathbf{P}\left(C_{1}\left(\mathbf{k}_{0}\right)\right)=\frac{N_{0}^{r}}{r ! N_{1}^{r-1} N_{2}} e^{-N_{0} / N_{1}}\left(1+O\left(\frac{\min \left(N_{0}, N_{1}\right)}{N_{2}}+\frac{1}{N_{0}}\right)\right) .
$$

С другой стороны, если $\mathbf{k} \in K(r) \backslash\left\{\mathbf{k}_{0}\right\}$, то $2 \leqslant k_{1}+\ldots+k_{r} \leqslant r$; учитывая (3) и (5), находим, что

$$
\begin{aligned}
\mathbf{P}\left(C_{1}(\mathbf{k})\right)= & \frac{N_{0}^{[r]} N_{1}^{\left[k_{1}+\ldots+k_{r}\right]}}{N_{1}^{r}(1 !)^{k_{1}} \ldots(r !)^{k_{r}} k_{1} ! \ldots k_{r} ! N_{2}^{k_{1}+\ldots+k_{r}}} \\
& \times\left(1-\frac{k_{1}+\ldots+k_{r}}{N_{1}}\right)^{N_{0}-r}\left(1-\frac{1}{N_{2}}\right)^{\bar{\mu}_{1}^{(1)}-k_{1}-\ldots-k_{r}} \\
\leqslant & \frac{N_{0}^{r}}{N_{2}^{k_{1}+\ldots+k_{r}} N_{1}^{r-k_{1}-\ldots-k_{r}}} e^{-\left(k_{1}+\ldots+k_{r}\right)\left(N_{0}-r\right) / N_{1}} \\
= & O\left(\frac{N_{0}^{r}}{N_{2}^{2} N_{1}^{r-2}} e^{-2 N_{0} / N_{1}}\right)=O\left(\frac{N_{1}}{N_{2}} \mathbf{P}\left(C_{1}\left(\mathbf{k}_{0}\right)\right)\right)
\end{aligned}
$$

и в силу конечности множества $K(r)$ при условиях $N_{0}=O\left(N_{1}\right), N_{1}=O\left(N_{2}\right)$

$$
\mathbf{E} \mu_{r}^{(2)}=N_{2} \mathbf{P}\left(\theta_{1}=1\right)=\frac{N_{0}^{r}}{r ! N_{1}^{r-1}} e^{-N_{0} / N_{1}}\left(1+O\left(\frac{N_{0}+N_{1}}{N_{2}}+\frac{1}{N_{0}}\right)\right) .
$$

Для доказательства второй формулы положим $\mathbf{k}_{1}=(r, 0, \ldots, 0)$; тогда

$$
\mathbf{P}\left(\theta_{1}=1\right)=\mathbf{P}\left(C_{1}\left(\mathbf{k}_{1}\right)\right)+\sum_{\mathbf{k} \in K(r) \backslash\left\{\mathbf{k}_{1}\right\}} \mathbf{P}\left(C_{1}(\mathbf{k})\right) .
$$

Первое слагаемое есть

$$
\mathbf{P}\left(C_{1}\left(\mathbf{k}_{1}\right)\right)=\frac{N_{0}^{r}}{r ! N_{2}^{r}}\left(1+O\left(\frac{N_{0}}{N_{2}}+\frac{N_{0}}{N_{1}}+\frac{1}{N_{0}}\right)\right) .
$$

Если $\mathbf{k} \in K(r) \backslash\left\{\mathbf{k}_{1}\right\}$, то $1 \leqslant k_{1}+\ldots+k_{r} \leqslant r-1$ и при $N_{2}=O\left(N_{1}\right)$

$$
\mathbf{P}\left(C_{1}(\mathbf{k})\right) \leqslant \frac{N_{0}^{r}}{N_{2}^{k_{1}+\ldots+k_{r}} N_{1}^{r-k_{1}-\ldots-k_{r}}} \leqslant \frac{N_{0}^{r}}{N_{1} N_{2}^{r-1}}=O\left(\frac{N_{2}}{N_{1}} \mathbf{P}\left(C_{1}\left(\mathbf{k}_{1}\right)\right)\right) .
$$

Так же, как в первом случае, из последних оценок с учетом соотношения $N_{0}=O\left(N_{2}\right)$ следует, что

$$
\mathbf{E} \mu_{r}^{(2)}=N_{2} \mathbf{P}\left(C_{1}\left(\mathbf{k}_{1}\right)\right)\left(1+O\left(\frac{N_{2}}{N_{1}}\right)\right)=\frac{N_{0}^{r}}{r ! N_{2}^{r-1}}\left(1+O\left(\frac{N_{0}}{N_{2}}+\frac{1}{N_{0}}+\frac{N_{2}}{N_{1}}\right)\right) .
$$

Тем самым лемма 1 доказана. 
Лемма 2. В равновероятной двухэтапной схеме размещения частич для любых $r \in$ $\{2,3, \ldots\}, m \in\{1,2, \ldots\} n p u N_{0}, N_{1}, N_{2} \rightarrow \infty, N_{0}=o\left(N_{2}\right), N_{0}=O\left(N_{1}\right)$

$$
\mathbf{E} \mu_{r}^{[m]}=\left(\mathbf{E} \mu_{r}\right)^{m}\left(1+O\left(\frac{N_{0}}{N_{2}}+\frac{1}{N_{0}}\right)\right) .
$$

Доказательство. Как в лемме 1,

$$
\begin{gathered}
\mathbf{E} \mu_{r}^{(2)}=N_{2} \mathbf{P}\left(\theta_{1}=1\right), \\
\mathbf{E}\left(\mu_{r}^{(2)}\right)^{[m]}=N_{2}^{[m]} \mathbf{P}\left(\theta_{1}=\ldots=\theta_{m}=1\right), \\
\mathbf{P}\left(\theta_{1}=1\right)=\sum_{\mathbf{k} \in K(r)} \mathbf{P}\left(C_{1}(\mathbf{k})\right), \\
\mathbf{P}\left(\theta_{1}=\ldots=\theta_{m}=1\right)=\sum_{\mathbf{k}_{1}, \ldots, \mathbf{k}_{m} \in K(r)} \mathbf{P}\left(C_{1}\left(\mathbf{k}_{1}\right) \ldots C_{m}\left(\mathbf{k}_{m}\right)\right) .
\end{gathered}
$$

Поэтому утверждение леммы следует из равенства

$$
\mathbf{P}\left(C_{1}\left(\mathbf{k}_{1}\right) \ldots C_{m}\left(\mathbf{k}_{m}\right)\right)=\mathbf{P}\left(C_{1}\left(\mathbf{k}_{1}\right)\right) \ldots \mathbf{P}\left(C_{m}\left(\mathbf{k}_{m}\right)\right)\left(1+O\left(\frac{N_{0}}{N_{2}}+\frac{1}{N_{0}}\right)\right),
$$

где $\mathbf{k}_{i}=\left(k_{i 1}, \ldots, k_{i r}\right), k_{i 1}+2 k_{i 2}+\ldots+r k_{i r}=r, i=1, \ldots, m$.

Докажем (6). Условная вероятность события $C_{1}\left(\mathbf{k}_{1}\right) \ldots C_{m}\left(\mathbf{k}_{m}\right)$ при условии, что $\mu_{1}=v_{1}, \ldots, \mu_{r}=v_{r}$, равна

$$
\frac{v_{1}^{\left[\varkappa_{1}\right]} \ldots v_{r}^{\left[\varkappa_{r}\right]}}{k_{11} ! \ldots k_{m r} ! N_{2}^{\chi_{1}+\ldots+x_{r}}}\left(1-\frac{m}{N_{2}}\right)^{\bar{\mu}_{1}^{(1)}-\varkappa_{1}-\ldots-\varkappa_{r}}
$$

где $\varkappa_{j}=k_{1 j}+\ldots+k_{m j}, j=1, \ldots, r$. Поэтому вероятность в левой части (6) равна

$$
\mathbf{E} \frac{\mu_{1}^{\left[\varkappa_{1}\right]} \ldots \mu_{r}^{\left[\chi_{r}\right]}}{k_{11} ! \ldots k_{m r} ! N_{2}^{\varkappa_{1}+\ldots+x_{r}}}\left(1-\frac{m}{N_{2}}\right)^{\bar{\mu}_{1}^{(1)}-\varkappa_{1}-\ldots-x_{r}} .
$$

Учитывая (4) и то, что $\min \left(N_{0}, N_{1}\right)=O\left(N_{0}\right)$ в силу условия $N_{0}=O\left(N_{1}\right)$, получаем:

$$
\mathbf{P}\left(C_{1}\left(\mathbf{k}_{1}\right) \ldots C_{m}\left(\mathbf{k}_{m}\right)\right)=\mathbf{E} \mu_{1}^{\left[x_{1}\right]} \ldots \mu_{r}^{\left[x_{r}\right]} \frac{1}{k_{11} ! \ldots k_{m r} ! N_{2}^{x_{1}+\ldots+x_{r}}}\left(1+O\left(\frac{N_{0}}{N_{2}}\right)\right) .
$$

Согласно [1], с. 45, при $\varkappa_{1}+\ldots+r \varkappa_{r}=r m$

$$
\mathbf{E} \mu_{1}^{\left[\varkappa_{1}\right]} \ldots \mu_{r}^{\left[\varkappa_{r}\right]}=\frac{N_{0}^{[r m]} N_{1}^{\left[\varkappa_{1}+\ldots+\varkappa_{r}\right]}}{N_{1}^{r m}(1 !)^{\varkappa_{1}} \ldots(r !)^{\varkappa_{r}}}\left(1-\frac{\varkappa_{1}+\ldots+\varkappa_{r}}{N_{1}}\right)^{N_{0}-r m} .
$$

Проводя те же оценки, что в лемме 1 , получаем соотношение

$$
\begin{aligned}
\mathbf{P}\left(C_{1}\left(\mathbf{k}_{1}\right) \ldots C_{m}\left(\mathbf{k}_{m}\right)\right)=\frac{N_{0}^{r m}}{k_{11} ! \ldots k_{m r} !} & N_{2}^{x_{1}+\ldots+x_{r}} N_{1}^{r m-x_{1}-\ldots-x_{r}}(1 !)^{\varkappa_{1}} \ldots(r !)^{\varkappa_{r}} \\
& \times e^{-\left(x_{1}+\ldots+x_{r}\right) N_{0} / N_{1}}\left(1+O\left(\frac{N_{0}}{N_{2}}+\frac{1}{N_{0}}\right)\right) .
\end{aligned}
$$


Аналогично преобразуем сомножители в правой части (6):

$$
\begin{aligned}
\mathbf{P}\left(C_{i}\left(\mathbf{k}_{i}\right)\right)= & \mathbf{E} \mu_{1}^{\left[k_{i 1}\right]} \ldots \mu_{r}^{\left[k_{i r}\right]} \frac{1}{k_{i 1} ! \ldots k_{i r} ! N_{2}^{k_{i 1}+\ldots+k_{i r}}}\left(1+O\left(\frac{N_{0}}{N_{2}}\right)\right) \\
= & \frac{N_{0}^{r}}{k_{i 1} ! \ldots k_{i r} ! N_{2}^{k_{i 1}+\ldots+k_{i r}} N_{1}^{r-k_{i 1}-\ldots-k_{i r}}(1 !)^{k_{i 1}} \ldots(r !)^{k_{i r}}} e^{-\left(k_{i 1}+\ldots+k_{i r}\right) N_{0} / N_{1}} \\
& \times\left(1+O\left(\frac{N_{0}}{N_{2}}+\frac{1}{N_{0}}\right)\right)
\end{aligned}
$$

Значит,

$$
\mathbf{P}\left(C_{1}\left(\mathbf{k}_{1}\right) \ldots C_{m}\left(\mathbf{k}_{m}\right)\right)=\mathbf{P}\left(C_{1}\left(\mathbf{k}_{1}\right)\right) \ldots \mathbf{P}\left(C_{m}\left(\mathbf{k}_{m}\right)\right)\left(1+O\left(\frac{N_{0}}{N_{2}}+\frac{1}{N_{0}}\right)\right) .
$$

Отметим, что постоянные в оценках равномерны по всем событиям, так как при фиксированных $r, m$ суммы по $\mathbf{k}_{1}, \ldots, \mathbf{k}_{m}$ состоят из конечного числа слагаемых. Лемма 2 доказана.

Доказательство теоремы 1. Из леммы 2 следует, что $\mathbf{E} \mu_{r}^{[m]} \rightarrow \lambda^{m}$ при условиях теоремы 1 , следовательно, распределение $\mu_{r}^{(2)}$ сходится к распределению Пуассона с параметром $\lambda$. Теорема 1 доказана.

\section{Список литературы}

1. Колчин В. Ф., Севастьянов Б. А., Чистяков В. П., Случайные размещения. Наука, Москва, 1976.

2. Зубков А. М., Шибанов О. К., Многоступенчатые схемы размещения частиц по ячейкам. Обозрение прикладной и промыиленной математики (2002) 9, №1, 115-116.

3. Зубков А. М., Шибанов О. К., Двухступенчатая схема размещения частиц по ячейкам. Обозрение прикладной и промыиленной математики (2002) 9, №2, 378-379.

Статья поступила 12.12.2005. 